\title{
Microspheres with Atomic-Scale Tolerances Generate Hyperdegeneracy
}

\author{
Jacob Kher-Alden $\odot,{ }^{1}$ Shai Maayani, ${ }^{1}$ Leopoldo L. Martin, ${ }^{2}$ Mark Douvidzon, ${ }^{1}$ Lev Deych, ${ }^{3,4}$ and Tal Carmon ${ }^{1, *}$ \\ ${ }^{1}$ Department of Mechanical Engineering, Technion-Israel Institute of Technology, 3200003 Haifa, Israel \\ ${ }^{2}$ Departamento de Fisica and IUdEA, Universidad de La Laguna (ULL) 38200, Tenerife, Spain \\ ${ }^{3}$ Physics Department, Queens College of CUNY, Flushing, New York 11367, USA \\ ${ }^{4}$ Graduate Center of CUNY, 365 5th Ave, New York 10016, USA
}

(Received 16 April 2020; accepted 17 July 2020; published 31 August 2020)

\begin{abstract}
Degeneracies play a crucial rule in precise scientific measurements as well as in sensing applications. Spherical resonators have a high degree of degeneracy thanks to their highest symmetry; yet, fabricating perfect spheres is challenging because even a stem to hold the sphere breaks the symmetry. Here we fabricate a levitating spherical resonator that is evanescently coupled to a standard optical fiber. We characterize the resonators to exhibit an optical quality factor exceeding a billion, $10 \mu \mathrm{m}$ radius, and sphericity to within less than $1 \AA$. Using our high quality and sphericity, we experimentally lift degeneracies of orders higher than 200, which we resolve with optical finesse exceeding 10000000 . We then present our experimentally measured degenerate modes as well as their density of states next to our corresponding theoretical calculation. Our contactless photonic resonator is compatible with standard telecom fiber technology, exhibits the highest resonance enhancement as defined by (quality factor)/(mode volume), and the modes populating our cavity show the highest order of degeneracy reported in any system ever studied. This is in comparison with other settings that typically utilize the lowest-order twofold degeneracy.
\end{abstract}

DOI: 10.1103/PhysRevX.10.031049

Degeneracies, including exceptional points [1-4], where level coalescence with only one eigenmode, play a crucial role in multiple fields ranging from sensing of nanoparticles, biological, and chemical analytes $[1,2,5-11]$ to gyroscopes and other navigation systems $[12,13]$. Often, optical resonators are being used to generate degeneracies that are typically twofold. The general problem of experimentally demonstrating the highest degree of degeneracy relates to the need for resonators to possess the highest degree of nontrivial symmetry (e.g., perfect spheres) - as theory suggests [14]. These resemble cases such as in cesium, where the spherically symmetric Coulomb potential supports hyperfine splitting. Yet, making such perfect spherical resonators, to host hyperdegeneracy, is impossible using current fabrication techniques or even 3D printers. This is because even a stem to hold the sphere breaks the symmetry. Here, we fabricate optically tweezed $[15,16]$ contactless resonators, spherical to within fabrication tolerances of less than the Bohr radius, in the vicinity of tapered-fiber couplers.

\footnotetext{
*Corresponding author. tcarmon@technion.ac.il

Published by the American Physical Society under the terms of the Creative Commons Attribution 4.0 International license. Further distribution of this work must maintain attribution to the author(s) and the published article's title, journal citation, and DOI.
}

Subject Areas: Optics, Photonics

Giving devices levity, while seemingly depriving them of their gravity [17] was always igniting the imagination. Such a levitation here permits almost perfect microspheres since in small droplets, surface tension dominates over gravity to minimize surface area toward the smallest possible area (per a given volume) that spheres exhibit. In this regard, the optical tweezers apply a force comparable to gravity in size, but in the opposite direction. Our vision includes better sensitivity using highest degeneracy orders, and scalability of the levitating resonator to optical circuits made of many levitators, by using currently available holographic-tweezers technology [1]. A longerterm vision relates to the fact that the degenerate modes here are orbital angular-momentum modes, which can be utilized to construct high-dimensional modes of photons called qudits [18]. Combining degenerate qudits with low absorption [19] might impact time-complexity reduction in problem solving [20]. In more detail, perturbed symmetry in nature is commonly related to degeneracy. For example, hyperfine splitting in alkali atoms represents degeneracy that is lifted by perturbations of the atom spherically symmetric Coulomb potential by merely intranucleus inhomogeneities. Inspired by this symmetry-degeneracy relation, we extend this principle from atoms to classicalwave resonators [14,21], with lifted degeneracies due to the perturbation by the tweezer pressure and gravity. Such resonators can host light, sound, or other waves in nature. Generally speaking, the highest nontrivial symmetry in 

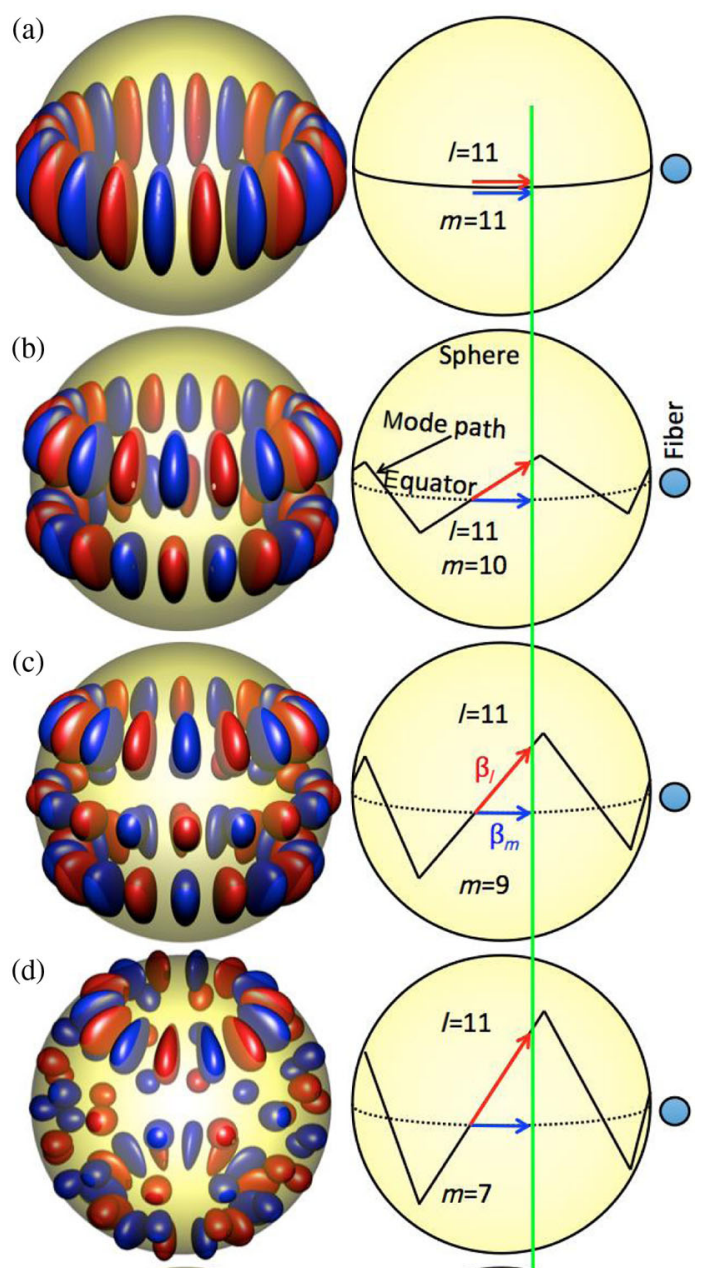

(e)
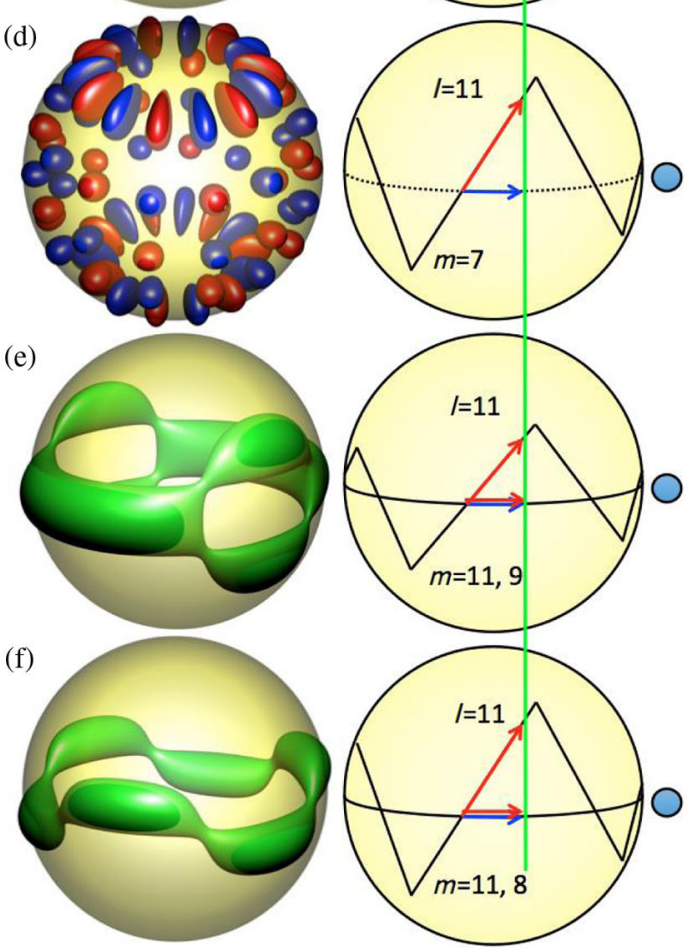

FIG. 1. Optical modes in spherical resonators. (a)-(d) High-degree degeneracy in a spherical resonator where a family of modes have the same propagation constant $\beta_{m}$ in the net direction of propagation (blue arrow), irrespective of their number of maxima between poles (here 1, 2, 3, and 5). (e),(f) Standing interference pattern generated by modes having the same resonance frequency, but with a different number of wavelengths along the equator $(m)$. Red (blue) surfaces were calculated with Eq. (SM1) of the Supplemental Material [25] to represent the location where the electric field falls to half of its maximum (minimum). Green represents an equal intensity surface. The polarization is TE, the radial number is 1 , the refractive index is 1.4 , and the sphere's circumference is 11 optical wavelengths. nature is spherical-invariance with respect to rotations by any angle around any axis passing through the center of the corresponding sphere. The highest symmetry of spheres results in modes with the highest degree of degeneracy. To give it a scale, the order of degeneracy in our microspheres is more than 100 times larger when compared to a ring. Realization of optical resonators with shapes only weakly deviating from spherical is of great interest. For example, as it has been shown in Ref. [22], the sensitivity of the optical spectra of WGM resonators to the presence of various analytes improves dramatically when deviation from sphericity of a resonator drops below a critical value [23]. Observation of spherical-symmetry degeneracy in microresonators is challenging due to fabrication difficulties. Even if planar lithography could produce smooth microspheres with a sufficiently small deviation from sphericity, their support will break the symmetry. We overcome this difficulty using optical levitation, which does not require any symmetry-breaking support. Contactless devices are also almost perfectly proof against contaminations, as demonstrated on silicon wafers [24], by keeping them cleanest and unscratched even at the atomic level. Levitating devices can hence benefit from both worlds-highest degree of cleanness to support lowest optical losses and symmetry to support highest degrees of degeneracy.

Optical resonances of spherical dielectrics [see Figs. 1(a)1(d) and Supplemental Material Sec. SM1 and Ref. [14] in [25]] are characterized by the number of wavelengths that can fit along the equator of a resonator $(l)$ and the number of the intensity maxima between their north and south pole $(l-m+1)$. For a given $l, m$ equals $-l,-l+1$, $-l+2, \ldots, l$, and the modes are therefore $2 l+1$ degenerate with respect to azimuthal (magnetic in the quantum context) number $m$. For instance, for a resonance characterized by $l=113$ one can expect 227 degenerate modes, which is typical to our experiment. Since eigenfrequencies of the degenerate modes are the same, they generate a standing interference structure, of the type that one can see in Figs. 1(e) and 1(f). This degeneracy can be qualitatively understood [14] by noting that modes having a smaller $m$ travel at larger inclinations with respect to the equator, which is compensated for by shorter circumference of circles lying above or below the equator [Figs. 1(a)-1(d), right]. This qualitative description is consistent with the characteristic equation for the frequencies of the resonances [23],

$$
n \frac{j_{l}^{\prime}(n k r)}{j_{l}(n k r)}=\frac{y_{l}^{\prime}(k r)}{y_{l}(k r)}
$$

where $r$ and $n$ are the sphere's radius and refractive index, $k$ is the wave vector, and $j_{l}(z)$ and $y_{l}(z)$ are the spherical Bessel functions of the first and second kind. Importantly, Eq. (1) does not contain $m$, making its solutions independent of this quantum number. When a spherical resonator is deformed into a spheroid, the degeneracy is lifted and new eigenfrequencies can be found at [23] 


$$
\nu_{l, m}=\nu_{l}^{(0)}-\frac{1}{3} \eta \nu_{l}^{(0)}\left(1-3 \frac{m^{2}}{l(l+1)}\right),
$$

where $\nu_{l}^{(0)}$ is the unperturbed frequency of the spherical resonator and $\eta$ is the shape deformation parameter defined in Supplemental Material, Sec. SM2 [25]. Such a split of the resonances was indeed experimentally observed in resonators with large deviations from sphericity $[21,28]$.

In our experiment (Fig. 2), we make droplets from silicone oil of $10 \mathrm{cSt}$ viscosity. It is helpful here that unlike solids, the liquid droplet does not contain any dislocation, inclinations, and thermally induced stresses, which are typical for solid resonators and reduce their optical quality [29]. The sphericity of the droplet, held by our tweezers, is perturbed by the combination of radiative pressure [30] and gravity.

In our experimental setup, a tunable laser is evanescently coupled via a tapered fiber [31] to an optically tweezed $[15,16]$ levitating droplet of silicone oil, and excites its resonances. This approach distinguishes our experiment from earlier works, in which resonances of levitating [16] or falling [32] droplets were excited by free-propagating light beams, where large phase mismatch could be solved by designing the cavity shape [33]. In our approach, phase matching is significantly improved by realizing contactless coupling to the standard fiber, tapered until its effective refractive index matches the resonator's effective index [31]. Resonances are observed as Lorentzian dips of the transmission through the fiber as we tune the frequency of the incident laser light.

Characterizing the linewidths of the dips reveals an optical quality factor of the resonances to be $1.2 \times 10^{9}$ with finesse of $11.6 \times 10^{6}$ [Fig. 3(e)]. Instead of a single resonance expected for an ideal sphere, experimental spectra exhibit multiple, sometimes partially overlapping resonances [Figs. 3(f)-3(j)]. When resonances overlap [Figs. 3(f), 3(i), and 3(j), orange lines] the incident light can excite simultaneously two modes, giving rise to a symmetric or asymmetric (with respect to the equatorial plane) intensity structures similar to the ones calculated in Figs. 1(e) and 1(f).

Analyzing the family of degenerate modes by their density of modes is expected to reveal that density goes down with frequency as theoretically derived in Eq. (SM12) [25]. Indeed, as one can qualitatively see [Figs. 4(a) and 4(b)], the resonances turn less dense as frequency goes up. Going now to a quantitative analysis, we fit our experimentally measured density of modes [Fig 4(c), blue] to its theoretically predicted [Fig 4(c), orange] value [Eq. (SM12)], using the spectral span of the hyperfine family $\Delta_{l}$ as a free parameter (Supplemental Material Sec. SM2 [25]). Our experimental parameters reflect angular momentum of $l=113$ and degeneracy order of 227. The theoretical model assumes that only one family of modes is presented in the frequency span that we analyze [Fig. 4(c)]. Yet, we expect that other

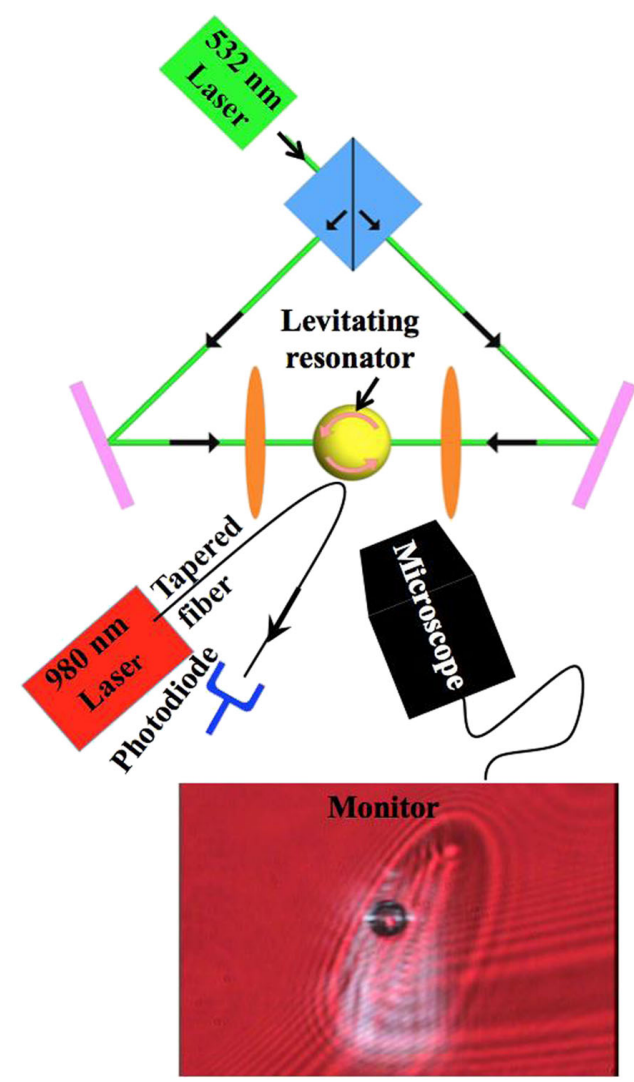

FIG. 2. Experimental setup for phase-matched coupling to a contactless sphere. A counterpropagating optical tweezer traps a silicone oil droplet. At the same time, a tapered-fiber coupler is brought nearby to the droplet. The transmission through the droplet resonator is monitored throughout the other side of the taper. The experiment is performed under the microscope while a monitor shows a micrograph of the droplet in which the bent tapered fiber is seen at the background, out of focus. The refractive index is 1.4 and the wavelength is $980 \mathrm{~nm}$.

families of modes, e.g., with higher radial order, might contribute stray modes with frequencies penetrating the spectral band that we measure. These stray modes are most likely to explain the fact that our measured density of mode [Fig. 4(c), blue] is typically above the corresponding theoretical model line that assumes one family of modes [Fig. 4(c), orange]. With this circumstance taken into account, we can conclude that the fit presented in Fig. 4(c) with $\Delta_{l}=1.66 \mathrm{GHz}$ agrees reasonably well with our experimental data. This value of $\Delta_{l}$ corresponds to the record low deformation parameter $\eta \simeq 5.42 \times 10^{-6}$, which translates into the horizontal stretch of the droplet diameter from its ideal sphere value by merely $0.98 \AA$. The evanescent coupling by the tapered fiber could also induce the perturbation to the eigenmodes and break the spherical symmetry of the system. Yet, calculating the taper's contribution to degeneracy in our case reveals that its effect is smaller by more than one order of magnitude, when compared to asphericity. 
(a)

(b)

(c)

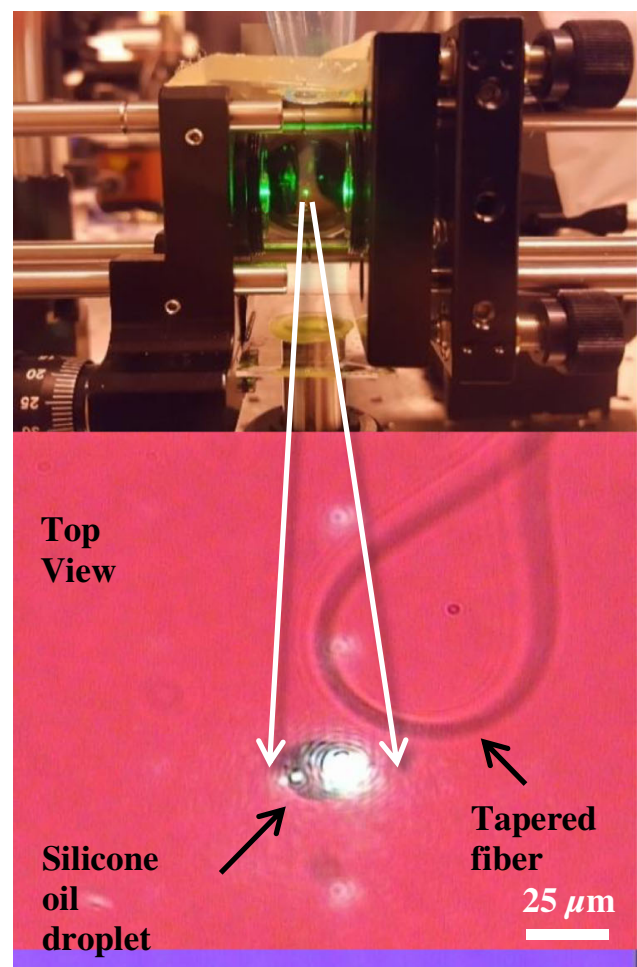

(d)

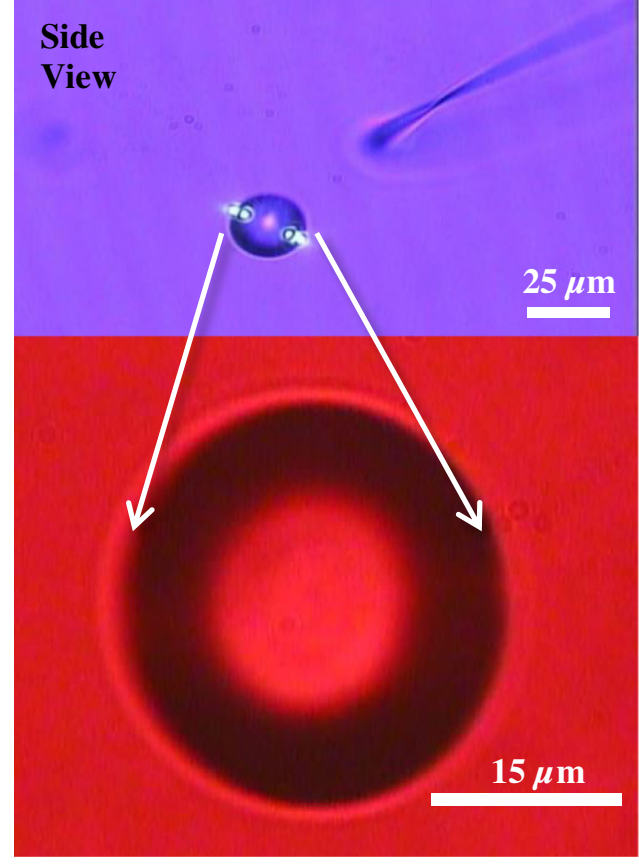

(e)

(f)
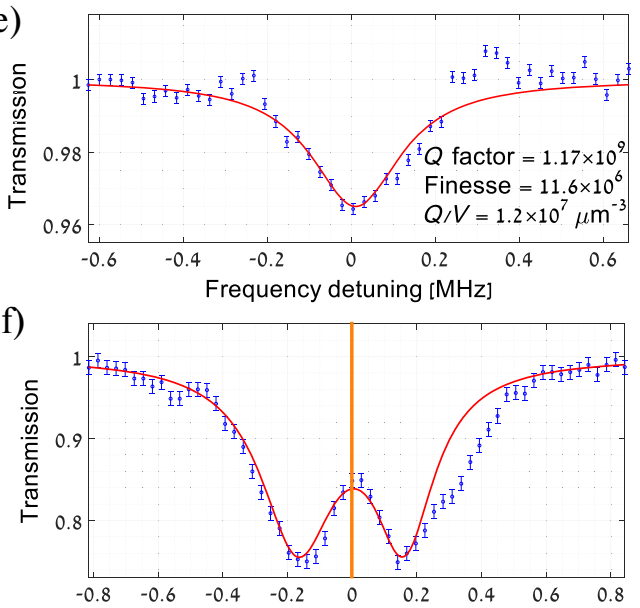

(g)

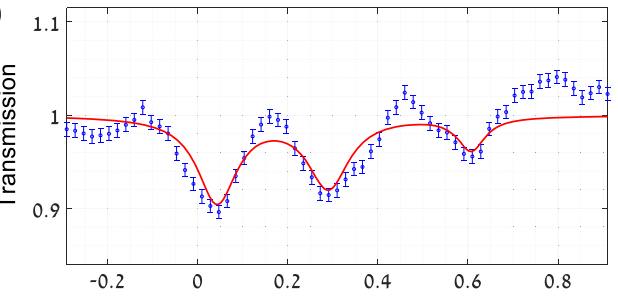

(h)

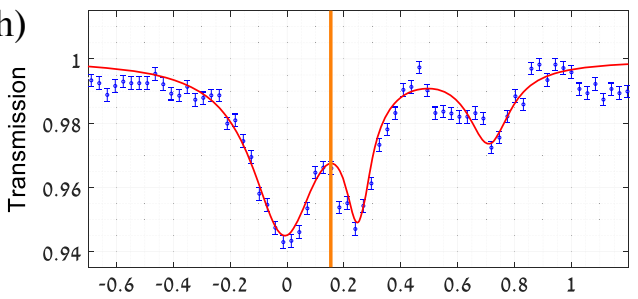

(i)

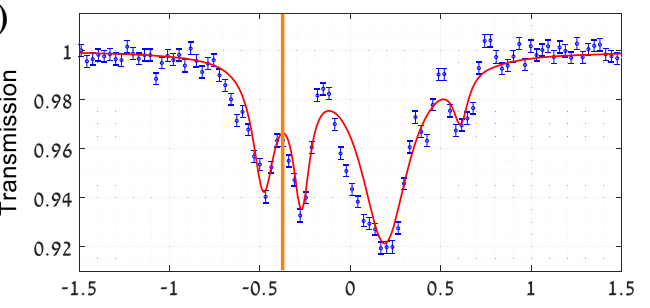

(j)

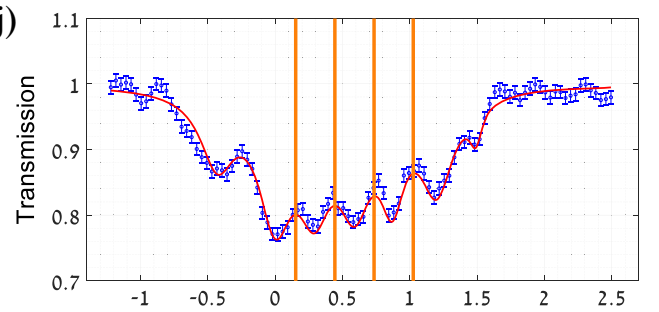

FIG. 3. A photograph of the experiment. (a) The levitating microdroplet is visible to the naked eye via scattering of the tweezers' green light. Micrographs of the experiment where we present top (b), side (c), and enlarged (d) views of the resonator-taper region. (e)-(j) Experimental results with 1-7 adjacent resonances (blue) together with a fit to a several-Lorentzians model (red). Resonances at (e)-(j) were measured at different spectral regions. The error bar represents the standard deviation. Orange lines represent regions of coresonating (partially overlapping) mode. $\lambda=980 \mathrm{~nm}, r=11.45 \mu \mathrm{m}$ for (d), (h), and (i), and $13.5 \mu \mathrm{m}$ for (f), (g), and (j), and $n=1.4$. Deviations of the measured transmission from the Lorentzian model relate to the nonperfectly uniform scanning rate of the tunable laser.

In conclusion, except for the hyperdegeneracy and ultrahigh finesse that levitators permit, our vision includes their scalability to optical circuits that will be made of many levitating devices. This can be done using the impressive boost in the resolution and refresh rate of a spatial-light modulator, suggesting that many optical devices, similar to the ones reported here, can levitate while one controls their positions [15] and shape [34]. In more detail, spatial-light 
(a)

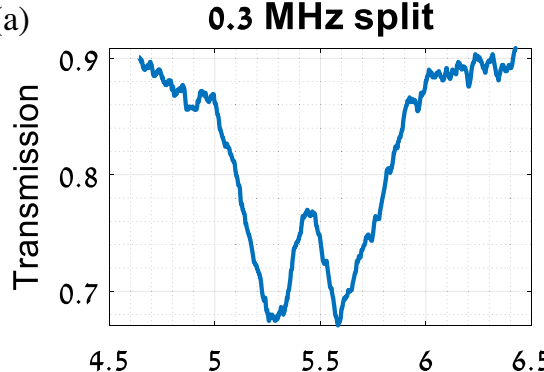

10 MHz split



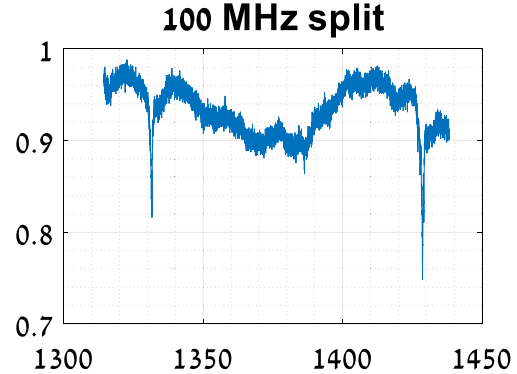

(b)

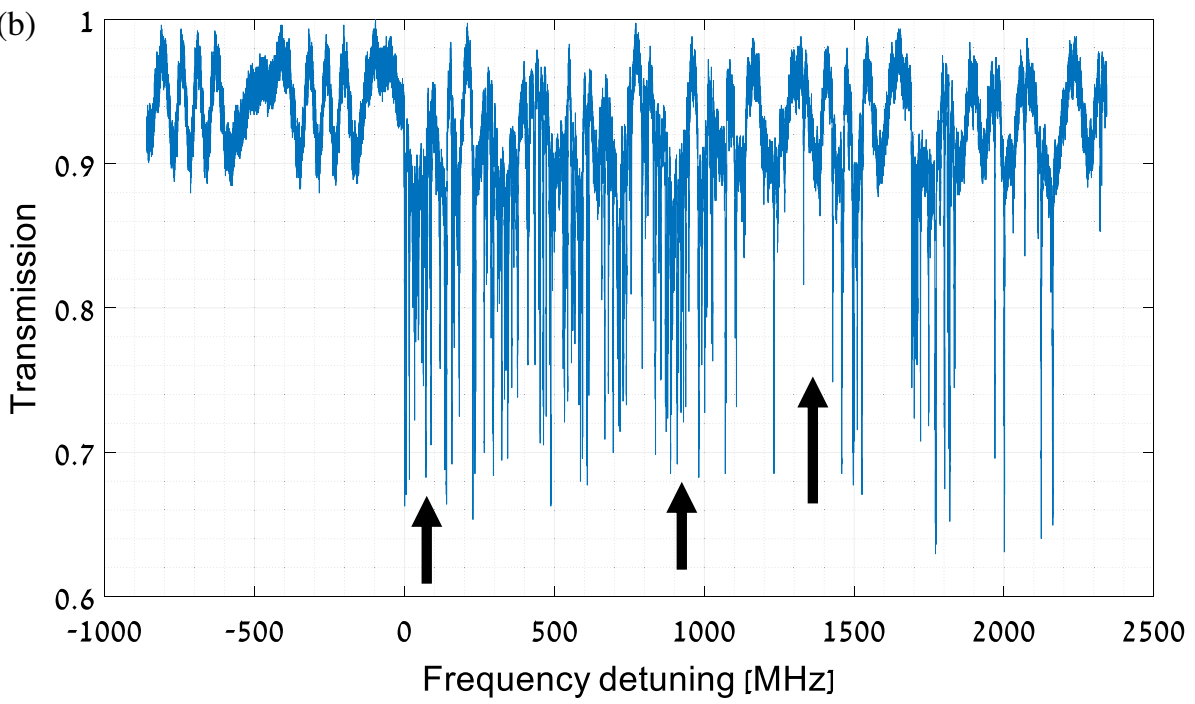

(c)

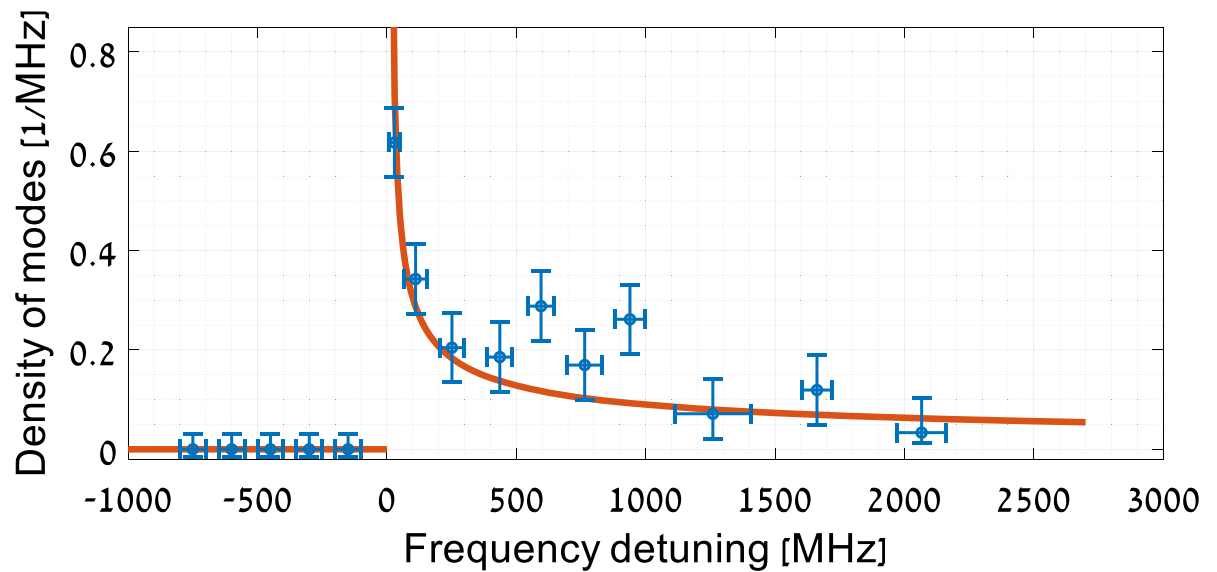

FIG. 4. Density of modes. The spectral transmission of the levitating resonator (a),(b) indicates no resonances at the left-hand side of (b), followed by dense resonances [(b), middle], and then fewer resonances per frequency span [(b), right-hand side]. Panel (a) represents an enlargement of spectral regions that are marked by an arrow in (b). (c) The experimentally measured density of modes (blue) as a function of the laser frequency, next to a theoretical fit using Eq. (SM12) [25] (orange). $\lambda=980 \mathrm{~nm}, r=13.5 \mu \mathrm{m}, n=1.4, l=113$, and degeneracy order is 227. Frequency error bars reflect standard deviation, and density-of-modes error bars reflect our estimation for fault counting of resonances. The irregular transmission background originates from fluctuations in the power of our pump laser.

modulators with $10 \times 10^{7}$ pixels are nowadays available, suggesting that levitators can be deformed upon request and then positioned by holographic tweezers, in 3D, upon need. In the past, using optical tweezers allowed deforming droplets to shapes such as triangles [34], suggesting that a variety of different devices might be possible. Continuously transforming multiple droplets, using dynamical traps, can give rise to photonics where the devices can also change while operating, a freedom that is challenging using current fabrication technologies. Such devices that change 
themselves upon need, while operating, are nowadays mainly common only in the art of science fiction, as appear in the movie Transformers.

We acknowledge help from Yehiel Mosses, Ariel Bar Yehuda, Moran Bercovici, Carmel Rotschild, and Eyal Zussman. This research was supported by the United States-Israel Binational Science Foundation (BSF) (Grant No. 2016670), by U.S. National Science Foundation (NSF) (Grant No. 1711801), by ICore: the Israeli Excellence center "Circle of Light" Grant No. 1902/12, and by the Israeli Science Foundation Grant No. 2013/15.

[1] H. Hodaei, A. U. Hassan, S. Wittek, H. Garcia-Gracia, R. El-Ganainy, D. N. Christodoulides, and M. Khajavikhan, Enhanced Sensitivity at Higher-Order Exceptional Points, Nature (London) 548, 187 (2017).

[2] W. Chen, Ş. K. Özdemir, G. Zhao, J. Wiersig, and L. Yang, Exceptional Points Enhance Sensing in an Optical Microcavity, Nature (London) 548, 192 (2017).

[3] M.-A. Miri and A. Alù, Exceptional Points in Optics and Photonics, Science 363, eaar7709 (2019).

[4] M. V. Berry, Physics of Nonhermitian Degeneracies, Czech. J. Phys. 54, 1039 (2004).

[5] F. Vollmer and S. Arnold, Whispering-Gallery-Mode Biosensing: Label-Free Detection Down to Single Molecules, Nat. Methods 5, 591 (2008).

[6] J. Zhu, S. K. Ozdemir, Y.-F. Xiao, L. Li, L. He, D.-R. Chen, and L. Yang, On-Chip Single Nanoparticle Detection and Sizing by Mode Splitting in an Ultrahigh-Q Microresonator, Nat. Photonics 4, 46 (2010).

[7] B.-B. Li, W. R. Clements, X.-C. Yu, K. Shi, Q. Gong, and Y.-F. Xiao, Single Nanoparticle Detection Using Split-Mode Microcavity Raman Lasers, Proc. Natl. Acad. Sci. U.S.A. 111, 14657 (2014).

[8] J. Wiersig, Enhancing the Sensitivity of Frequency and Energy Splitting Detection by Using Exceptional Points: Application to Microcavity Sensors for Single-Particle Detection, Phys. Rev. Lett. 112, 203901 (2014).

[9] X. Fan and I. M. White, Optofluidic Microsystems for Chemical and Biological Analysis, Nat. Photonics 5, 591 (2011).

[10] N. Zhang, S. Liu, K. Wang, Z. Gu, M. Li, N. Yi, S. Xiao, and Q. Song, Single Nanoparticle Detection Using Far-Field Emission of Photonic Molecule Around the Exceptional Point, Sci. Rep. 5, 11912 (2015).

[11] I. M. White, H. Oveys, and X. Fan, Liquid-Core Optical Ring-Resonator Sensors, Opt. Lett. 31, 1319 (2006).

[12] Y.-H. Lai, Y.-K. Lu, M.-G. Suh, and K. Vahala, Observation of the Exceptional-Point-Enhanced Sagnac Effect, Nature (London) 576, 65 (2019).

[13] J. Ren, G. Harari, A. U. Hassan, W. Chow, M. Soltani, M. P. Hokmabadi, D. Christodoulides, and M. Khajavikhan, PT-Symmetric Microring Laser Gyroscope, in Proceedings of the Conference on Lasers and Electro-Optics, 2018, FM4E.5, https://www.osapublishing.org/abstract.cfm?uri= CLEO_QELS-2018-FM4E.5, https://par.nsf.gov/servlets/ purl/10077418.
[14] B. E. Little, J. P. Laine, and H. A. Haus, Analytic Theory of Coupling from Tapered Fibers and Half-Blocks into Microsphere Resonators, J. Lightwave Technol. 17, 704 (1999).

[15] D. G. Grier, A Revolution in Optical Manipulation, Nature (London) 424, 810 (2003).

[16] A. Ashkin and J. M. Dziedzic, Observation of Resonances in the Radiation Pressure on Dielectric Spheres, Phys. Rev. Lett. 38, 1351 (1977).

[17] B. Franklin, The Private Correspondence of Benjamin Franklin, Letters on miscellaneous subjects (1818); https:// www.google.com/books?hl=en\&lr=\&id=joDSUISyUToC $\&$ oi $=$ fnd $\& p g=P R 1 \& d q=\% 22 \mathrm{We}+$ may + perhaps + learn + to + deprive + large + masses $\% 22 \&$ ots $=-\mathrm{O}-\mathrm{D} 6 \mathrm{BJHJb} \& \operatorname{sig}=\mathrm{LOh} 7$ 3iOdS8DZ1d0IJ5tf__HySYo

[18] M. Erhard, R. Fickler, M. Krenn, and A. Zeilinger, Twisted Photons: New Quantum Perspectives in High Dimensions, Light Sci. Appl. 7, 17146 (2018).

[19] J. Leach, B. Jack, J. Romero, A. K. Jha, A. M. Yao, S. Franke-Arnold, D. G. Ireland, R. W. Boyd, S. M. Barnett, and M. J. Padgett, Quantum Correlations in Optical AngleOrbital Angular Momentum Variables, Science 329, 662 (2010).

[20] L. K. Grover, A Fast Quantum Mechanical Algorithm for Database Search, in Proceedings of the Twenty-Eighth Annual ACM Symposium on Theory of Computing, 1996 (STOC '96) (Lov K Grover, 1996), p. 212, https://doi.org/ 10.1145/237814.237866.

[21] M. Hossein-Zadeh and K. J. Vahala, Fiber-Taper Coupling to Whispering-Gallery Modes of Fluidic Resonators Embedded in a Liquid Medium, Opt. Express 14, 10800 (2006).

[22] V. Shuvayev and L. Deych, Ab Initio Computational Analysis of Spectral Properties of Dielectric Spheroidal Resonators Interacting with a Subwavelength Nanoparticle, Phys. Rev. E 99, 013310 (2019).

[23] H. M. Lai, P. T. Leung, K. Young, P. W. Barber, and S. C. Hill, Time-Independent Perturbation for Leaking Electromagnetic Modes in Open Systems with Application to Resonances in Microdroplets, Phys. Rev. A 41, 5187 (1990).

[24] V. Vandaele, P. Lambert, and A. Delchambre, Non-Contact Handling in Microassembly: Acoustical Levitation, Precis. Eng. 29, 491 (2005).

[25] See Supplemental Material at http://link.aps.org/ supplemental/10.1103/PhysRevX.10.031049 for the dielectric sphere optical modes and calculating the density of modes for a mode family, which includes Refs. [26,27].

[26] M. I. Mishchenko, L. D. Travis, and A. A. Lacis, Scattering, Absorption, and Emission of Light by Small Particles (Cambridge University Press, Cambridge, New York, 2002).

[27] C. C. Lam, P. T. Leung, and K. Young, Explicit Asymptotic Formulas for the Positions, Widths, and Strengths of Resonances in Mie Scattering, J. Opt. Soc. Am. B 9, 1585 (1992).

[28] D. Keng, X. Tan, and S. Arnold, Whispering Gallery MicroGlobal Positioning System for Nanoparticle Sizing in Real Time, Appl. Phys. Lett. 105, 071105 (2014).

[29] M. L. Gorodetsky, A. A. Savchenkov, and V. S. Ilchenko, Ultimate $Q$ of Optical Microsphere Resonators, Opt. Lett. 21, 453 (1996). 
[30] P. C. F. Møller and L. B. Oddershede, Quantification of Droplet Deformation by Electromagnetic Trapping, Europhys. Lett. 88, 48005 (2009).

[31] J. C. Knight, G. Cheung, F. Jacques, and T. A. Birks, PhaseMatched Excitation of Whispering-Gallery-Mode Resonances by a Fiber Taper, Opt. Lett. 22, 1129 (1997).

[32] R. K. Chang and A. J. Campillo, Optical Processes in Microcavities (World Scientific, Singapore, 1996).
[33] X. Jiang, L. Shao, S. X. Zhang, X. Yi, J. Wiersig, L. Wang, Q. Gong, M. Loncar, L. Yang, and Y. F. Xiao, ChaosAssisted Broadband Momentum Transformation in Optical Microresonators, Science 358, 344 (2017).

[34] A. D. Ward, M. G. Berry, C. D. Mellor, and C. D. Bain, Optical Sculpture: Controlled Deformation of Emulsion Droplets with Ultralow Interfacial Tensions Using Optical Tweezers, Chem. Commun. 4515 (2006). 